\title{
Proteomic profiling of cisplatin-resistant and cisplatin-sensitive germ cell tumour cell lines using quantitative mass spectrometry
}

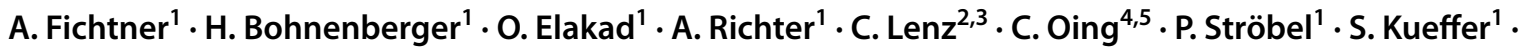 \\ D. Nettersheim ${ }^{6} \cdot$ F. Bremmer ${ }^{1}$ (i)
}

Received: 30 April 2021 / Accepted: 7 January 2022 / Published online: 27 January 2022

(c) The Author(s) 2022

\begin{abstract}
Purpose Advanced testicular germ cell tumours (GCT) generally have a good prognosis owing to their unique sensitivity towards cisplatin-based chemotherapies. However, cisplatin-resistant GCT have a poor outcome. Further studies are mandatory to better understand resistance mechanisms and develop therapeutic strategies for refractory GCTs.

Methods Protein levels in cisplatin-resistant GCT cell lines of NTERA-2, NCCIT and 2102EP were analyzed by quantitative proteomic mass spectrometry (MS) in combination with stable isotope labelling by amino acids in cell culture (SILAC). Differentially abundant protein markers of acquired cisplatin resistance were validated by Western blotting. Comprehensive bioinformatical annotation using gene set enrichment analyses (GSEA) and STRING interaction analysis were performed to identify commonly affected pathways in cisplatin resistance and the data were compared to the GCT cohort of the 'The Cancer Genome Atlas'.

Results A total of 4375 proteins were quantified by MS, 144 of which were found to be differentially abundant between isogenic resistant and sensitive cell line pairs (24 proteins for NTERA-2, 60 proteins for NCCIT, 75 proteins for 2102EP). Western blotting confirmed regulation of key resistance-associated proteins (CBS, ANXA1, LDHA, CTH, FDXR). GSEA revealed a statistically significant enrichment of DNA repair-associated proteins in all three resistant cell lines and specific additional processes for individual cell lines.

Conclusion High resolution MS combined with SILAC is a powerful tool and 144 significantly deregulated proteins were found in cisplatin-resistant GCT cell lines. Our study provides the largest proteomic in vitro library for cisplatin resistance in GCT, yet, enabling further studies to develop new treatment options for patients with refractory GCT.
\end{abstract}

Keywords Germ cell tumour $\cdot$ Cisplatin resistance $\cdot$ Proteomic analysis $\cdot$ Mass spectrometry $\cdot$ SILAC

\section{Introduction}

Testicular germ cell tumours (GCTs) are the most common malignant solid neoplasms in men between the age of 15 and 40 with an increasing incidence seen over the last 4 decades [1]. Histologically, type II GCT are divided into seminomas (SEM) and nonseminomatous GCT (NSGCT) according to the WHO [2]. NSGCT comprise distinct subentities, e.g. embryonal carcinomas (EC), yolk sac tumours,

A. Fichtner, H. Bohnenberger, S. Kueffer, D. Nettersheim, F. Bremmer were contributed equally.

\section{F. Bremmer}

felix.bremmer@med.uni-goettingen.de

Extended author information available on the last page of the article choriocarcinomas and teratomas (TER) [3]. The distinction between SEM and NSGCT is important because of varying treatment approaches, treatment responses and patients' prognosis [4]. The treatment of GCTs primarily comprises a radical inguinal orchiectomy of the affected testis. Subsequent cisplatin-based combination chemotherapy is required in metastatic GCTs [5, 6]. The introduction of cisplatinbased combination chemotherapy has led to cure rates of up to $90 \%$ even in metastatic disease stages [7-9]. Teratomas are of particular interest, as they are uniformly cisplatinresistant and surgery is the only successful therapy [6]. The number of patients with recurring disease that finally fail several lines of platinum-based chemotherapy is about $3-5 \%$ of all GCT patients and about $15 \%$ of patients with primary metastatic disease $[5,8,10,11]$, but they have an 
exceptionally poor prognosis with a life expectancy of only a few months [12].

In this study, we compared cisplatin-resistant and cisplatin-sensitive cell lineages of pluripotent NTERA-2 and NCCIT as well as the nullipotent 2102EP cells (with NCCIT deriving from a TP53 mutated mixed mediastinal GCT) [13, 14] by high-resolution mass spectrometry (MS) combined with stable isotope labelling with amino acids in cell culture (SILAC) [15-18]. The NTERA-2 and NCCIT cell lines represent EC, which can develop in all other types of NSGCT. Both cell lines represent a very well-studied in vitro model for NSGCTs. 2102EP represents an undifferentiated nullipotent EC, i.e. expresses markers of pluripotency, but do not tend to differentiate in response to differentiation-inducing signals. Cell lines representing seminoma were not used. We provide a proteomic resource library and functional annotations determined by gene set enrichment analyses (GSEA), the STRING algorithm and DAVID Gene Ontology annotation of acquired cisplatin resistance in GCT cell lines and compared our findings to the 'The Cancer Genome Atlas' (TCGA) cohort of 'testicular germ cell tumours'. These findings may help to detect new treatment options for GCT patients with cisplatin-resistant disease course. There are already some treatment options available, but as common mutations (e.g. receptors, kinases, etc.) are lacking in GCT, targetable mechanisms of cisplatin resistance need to be discovered and further analysed.

\section{Material and methods}

\section{Culture of human GCT cell lines}

The two human GCT cell lines NTERA-2 (CRL 1973) and NCCIT (CRL 2073) were supplied by ATCC, USA. 2102EP cells were a kind gift from PD Dr. Dr. Friedemann Honecker. All cell lines were cultured in HEPES-buffered RPMI-1640 (Biochrom, Berlin, Germany) supplemented with fetal calf serum (FCS, 10\%; CC Pro, Neustadt, Germany), penicillin (100 IU/ml; Sigma-Aldrich, Munich, Germany), streptomycin $(100 \mu \mathrm{g} / \mathrm{ml}$; Sigma-Aldrich) and L-glutamine (2 mM; Biochrom, Berlin, Germany). The incubation temperature was $37^{\circ} \mathrm{C}$ in a humid atmosphere with $5 \%$ carbon dioxide in the air.

\section{Induction of cisplatin resistance in human GCT cell lines}

For all parental GCT cell lines (NTERA-2, NCCIT, 2102EP), isogenic cisplatin-resistant sublines (NTERA-R, NCCIT-R, 2102EP-R) were established through repeated cisplatin-exposure to increasing sublethal cisplatin concentrations $(0.01-0.5 \mu \mathrm{g} / \mathrm{ml})$ over a time period of $9-12$ months, as described previously [19]. Cisplatin resistance was validated by treating sensitive and resistant cell lines after $72 \mathrm{~h}$ with increasing concentrations of cisplatin followed by comparing cellular viability using the CellTiter $96 \mathrm{R}$ AQ One Solution Cell Proliferation Assay (Promega GmbH, Waldorf, Germany) (Fig. 1a).

\section{Proteomic analysis of human GCT cell lines}

We performed protein expression profiling by mass spectrometry in combination with stable isotope labelling by amino acids in cell culture (SILAC) as described before [15-18]. NTERA-2, NCCIT and 2102EP cells and their resistant counterparts NTERA-2-R, NCCIT-R and 2102EP-R were cultured in RPMI 1640 medium supplemented with $10 \%$ dialyzed FCS (Invitrogen, Thermo Fisher GmbH, Bremen, Germany), $4 \mathrm{mM}$ glutamine, antibiotics and $0.115 \mathrm{mM}$ L-arginine $-{ }^{13} \mathrm{C}_{6}(\mathrm{Arg}+6)$ and $0.275 \mathrm{mM}$ L-lysine- ${ }^{2} \mathrm{D}_{4}$ (Lys +4$)$ or equimolar amounts of L-arginine${ }^{13} \mathrm{C}_{6}{ }^{15} \mathrm{~N}_{4}(\mathrm{Arg}+10)$ and $0.275 \mathrm{mM}$ L-lysine- ${ }^{13} \mathrm{C}_{6},{ }^{15} \mathrm{~N}_{2}$-Lys (Lys +8 ) (Eurisotop GmbH, Saarbrücken, Germany) for at least ten cell cycles. Labelled cells were lysed in $0.5 \%$ Nonidet P-40 buffer containing $50 \mathrm{mM}$ Tris/HCl, pH 7.8, $150 \mathrm{mM}$ $\mathrm{NaCl}, 1 \mathrm{mM} \mathrm{Na}_{3} \mathrm{VO}_{4}, 1 \mathrm{mM} \mathrm{NaF}, 0.2 \%$ lauryl maltoside and protease inhibitors (cOmplete ${ }^{\mathrm{TM}}$ Protease Inhibitor Cocktail, Roche, Mannheim, Germany). After isolation, the protein amounts were determined by a colorimetric BCA assay. Equal amounts of SILAC-labelled proteins (50 $\mu \mathrm{g}$ for each trial), were mixed and separated by SDS-PAGE (4-12\% NuPAGE Bis-Tris Gel, Invitrogen, Thermo Fisher Scientific $\mathrm{GmbH}$, Germany). Proteins were visualized with Coomassie brilliant blue stain and each lane cut into 11 equidistant slices irrespective of staining. Gel slices were reduced with $10 \mathrm{mM}$ DTT for $55 \mathrm{~min}$ at $56{ }^{\circ} \mathrm{C}$, alkylated with $55 \mathrm{mM}$ IAA for $20 \mathrm{~min}$ at $26^{\circ} \mathrm{C}$ and digested with modified trypsin (Promega GmbH, Walldorf, Germany) overnight at $37^{\circ} \mathrm{C}$. Resulting peptides were concentrated on a $\mathrm{C} 18$ precolumn $(25 \mathrm{~mm} \times 150 \mu \mathrm{m}$ I.D., Reprosil-Pur C18-AQ $120 \AA 5 \mu \mathrm{m}$, Dr. Maisch HPLC GmbH, Ammerbuch, Germany) for 5 min at a flow rate of $10 \mu \mathrm{l} / \mathrm{min}$ and separated on a C18 capillary column $(200 \mathrm{~mm} \times 75 \mu \mathrm{m}$ I.D., Reprosil-Pur C18-AQ $120 \AA$ $3 \mu \mathrm{m}$, Dr. Maisch HPLC GmbH, Ammerbuch, Germany) at a flow rate of $300 \mathrm{nl} / \mathrm{min}$, with a gradient of $5-35 \%$ acetonitrile $0.1 \%$ formic acid over 90 min using a Proxeon nano LC coupled to a Q Exactive mass spectrometer (Thermo Fisher Scientific GmbH, Bremen, Germany). MS conditions were as follows: spray voltage, $1.8 \mathrm{kV}$; heated capillary temperature, $270{ }^{\circ} \mathrm{C}$; and normalized collision-energy (NCE) 28. The mass spectrometer was operated in data-dependent acquisition mode. Survey MS spectra were acquired in the Orbitrap $(\mathrm{m} / \mathrm{z} 350-1600)$ with a resolution setting of 70,000 at $\mathrm{m} / \mathrm{z} 200$, a target fill value of $1 \mathrm{e} 6$ and a maximum fill time of $60 \mathrm{~ms}$. The 15 most intense ions were sequentially 

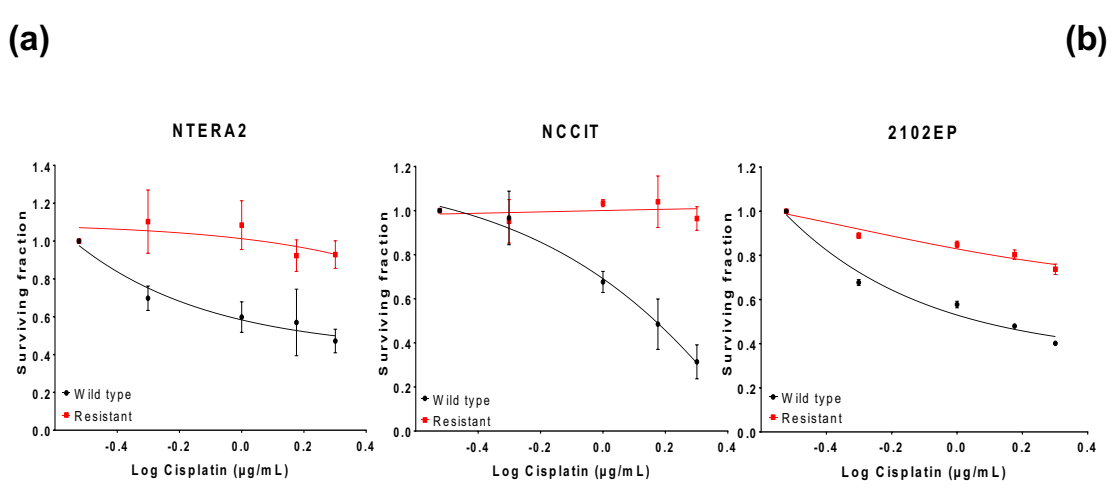

(b)

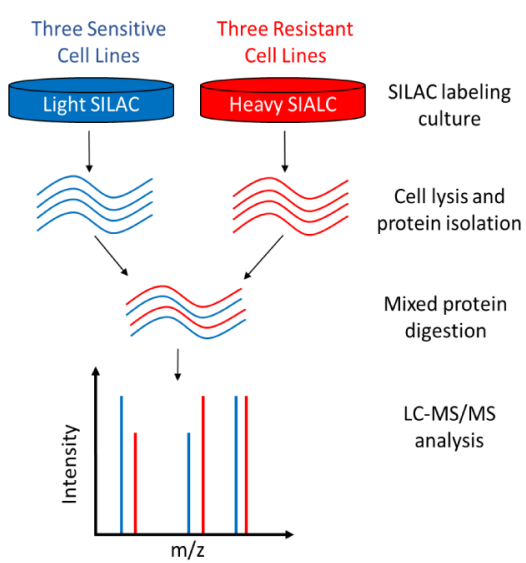

(c)

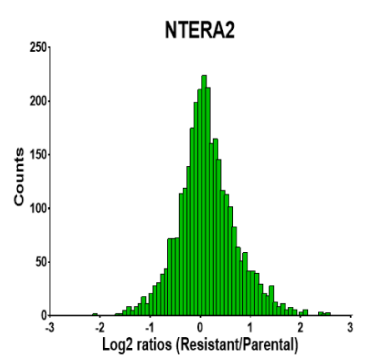

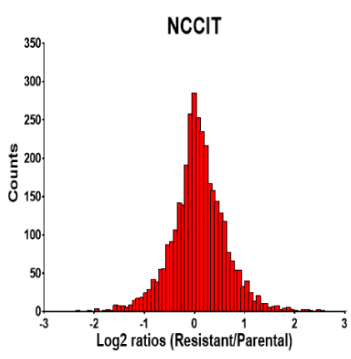

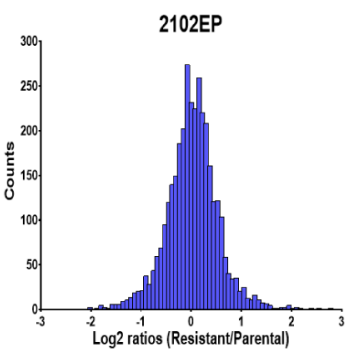

(d)

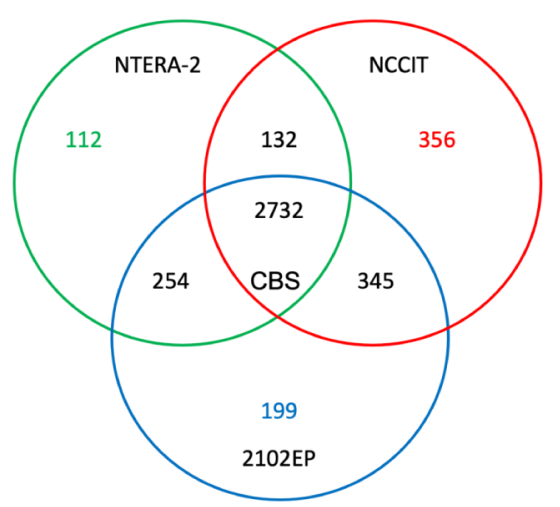

(f)

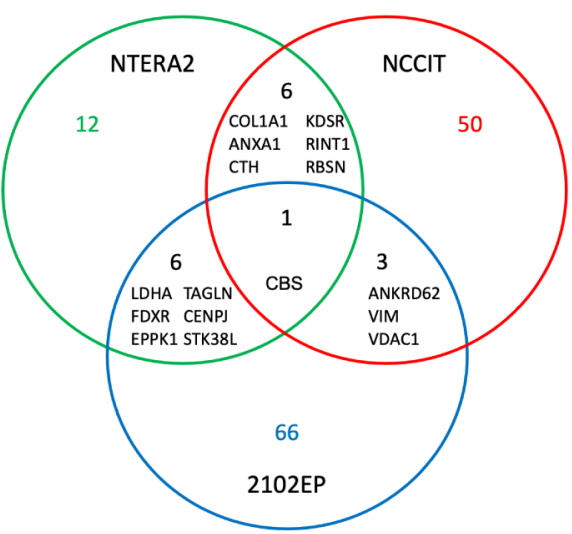

Fig. 1 Proteomic comparison of cisplatin-resistant and cisplatinsensitive GCT cell lines. a Comparison of cellular viability with increasing cisplatin concentrations in cultures of cisplatin-sensitive and cisplatin-resistant cell lines of NTERA-2, NCCIT and 2102EP with significant higher viability in resistant cell lines with high concentrations of cisplatin. b The diagram demonstrates the workflow of the three cisplatin-resistant and three cisplatin-sensitive cell lines

isolated for HCD MS/MS fragmentation and detection at a resolution setting of 17,500, a target fill value of 2e5 and a maximum fill time of $60 \mathrm{~ms}$. Raw data were analysed with for SILAC labelling and LC-MS/MS analysis. c Normal distribution of $\log _{2}$ SILAC ratios among the three named cell lines was seen. d Numeric Venn diagram of all quantified proteins in the three cell lines NTERA-2, NCCIT and 2102EP. e Principle component analysis showing technical and biological reproducibility of proteomic analysis $\mathbf{f}$ Numeric Venn diagram of significantly differentially regulated proteins in all three cell lines

MaxQuant software version 1.3.0.5 (Max Planck Institute for Biochemistry, Martinsried, Germany) against the UniprotKB human reference proteome revision 02-2017 containing 
92,928 sequence entries. Up to two missed cleavages of trypsin were allowed. Oxidized methionine, $\mathrm{N}$-terminal protein acetylation and the respective isotope-labelled arginine and lysine residues were searched as variable modifications and cysteine carbamidomethylation as fixed modification. The false discovery rates at the protein and peptide levels were both set to $1 \%$, respectively. Missing values in individual experiments are denoted by 'Not a Number $(\mathrm{NaN})$ '. No further data imputation or similar was used.

\section{Statistical analysis}

Perseus Software version 1.5.2.6 (Max Planck Institute for Biochemistry, Martinsried, Germany) was used for statistical evaluation. The mean of the biological replicates was calculated for each cell line (mean resistant/parental ratio). In order to compare $\log _{2}$ ratios of quantification to the intensity, $\log _{10}$ values were calculated for intensities of each mean value. Afterwards, significance $B$ analysis using a Wilcoxon-Mann-Whitney test with a Benjamini-Hochberg FDR $<5 \%$ was performed by Perseus software to calculate the $p$ values of outlier proteins from the 1/1 ratio [20]. $\log _{10}$ intensity was plotted against $\log _{2}$ ratios of quantified resistant/parental ratios. Proteins were coloured according to their $p$ values where blue colour means $>0.05$, red color $0.05-0.01$, yellow between 0.01 and 0.001 and light green coloured proteins mean that they have $p$ values less than 0.001 .

\section{Western blot analysis}

Cell lines were lysed in RIPA buffer (1 1 PBS Dulbecco pH 7.4; 5 g 5\% sodium deoxycholate, $10 \mathrm{ml}$ IGEPAL ${ }^{\circledR}$ CA-630) with protease inhibitors. Protein concentration was quantified by the Bio-Rad DC Protein Assay (Bio-Rad Laboratories $\mathrm{GmbH}$, Feldkirchen, Germany). The Western blot analyses were performed using the following primary antibody dilutions: monoclonal rabbit anti-CBS (D8F2P, Cell Signalling Technology ${ }^{\circledR}$, Massachusetts, USA, 1:1000), polyclonal rabbit anti-LDHA (Cell Signalling Technology ${ }^{\circledR}$, Massachusetts, USA, 1:1000), monoclonal rabbit anti-ANXA1 (D16A10, Cell Signalling Technology ${ }^{\circledR}$, Massachusetts, USA, 1:1000), monoclonal rabbit anti-CTH (D4E9J, Cell Signalling Technology ${ }^{\circledR}$, Massachusetts, USA, 1:1000) monoclonal mouse anti FDXR (6C2, Invitrogen, Thermo Fisher Scientific, Darmstadt, Germany 1:1000). Polyclonal immunoglobulins/HRP secondary antibodies (1:1000, Dako, Agilent Technologies GmbH, Waldbronn, Germany) were used for the detection of primary antibodies. Membranes were developed using the ECL system (Amersham Bioscience Europe GmbH, Freiburg, Germany).

\section{Gene set enrichment analysis}

Functional annotations were predicted using gene set enrichment analysis (GSEA) software downloaded from the home page (http://www.gsea-msigdb.org/gsea/index. jsp) $[21,22] . \log 2$ transformed proteomics resistance vs native ratios and the value 0 as native pendant were subjected uploaded into the software. Enrichment analysis was performed on the HALLMARK GeneSets with 1000 permutations on the original dataset format and gene set as Permutation type. Significant enrichments were defined as nominal $p$ values $<0.05$ and FDR $q<25 \%$ as suggested by GSEA. For visualization in a bar graph $p$ - and $q$-values were $-\log 2$ transformed. Enrichment plots were saved from the GSEA reports.

\section{Online analyses tools and software}

The STRING algorithm was used to predict protein-protein interaction by confidence using standard settings (https://string-db.org) [23]. The DAVID annotation has been used to predict molecular functions of deregulated genes or proteins found in mass spectrometry analyses based on 'Gene Ontology' (GO), 'Kyoto Encyclopedia of Genes and Genomes' (KEGG), Uniprot and INTEPRO (https://david.ncifcrf.gov/home.jsp) [24]. The proteins detected in this study were also compared with the TCGA dataset of 'testicular germ cell tumours' via cBioPortal (https://www.cbioportal.org/) [25, 26].

\section{Results}

\section{Quantitative proteomic profiling of cisplatin-resistant human GCT cell lines}

We compared the protein profiles of three cisplatinsensitive human GCT cell lines to their complementary cisplatin-resistant subclones using high resolution mass spectrometry in combination with SILAC (Fig. 1b). Normal Gaussian distribution of $\log _{2}$ SILAC ratios were seen in all three cell lines (Fig. 1c, Supplementary Fig. 1). In total, 4375 proteins were detected (Fig. 1d, Supplementary Table 1). Principle component analysis demonstrated high technical and biological reproducibility of proteomic analysis (Fig. 1e). A significantly different regulation between resistant and sensitive cell lines was found for 25 proteins in NTERA-2, for 60 proteins in NCCIT and for 75 proteins in 2102EP (Fig. 1e and f, 2a-c). Of these, 16 were significantly deregulated in at least two of the three tested cell lines (Table 1). 
(a)

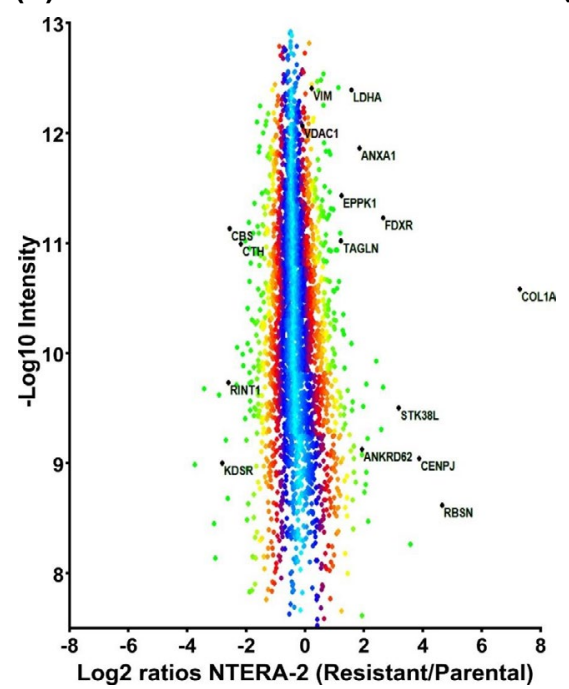

(b)

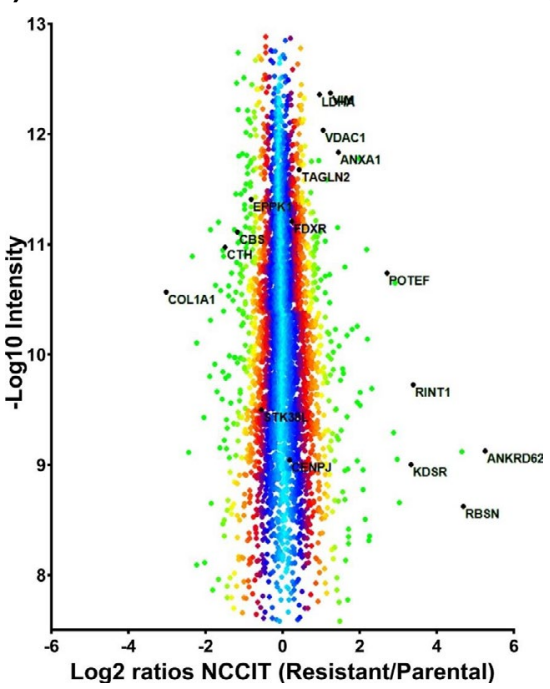

(c)

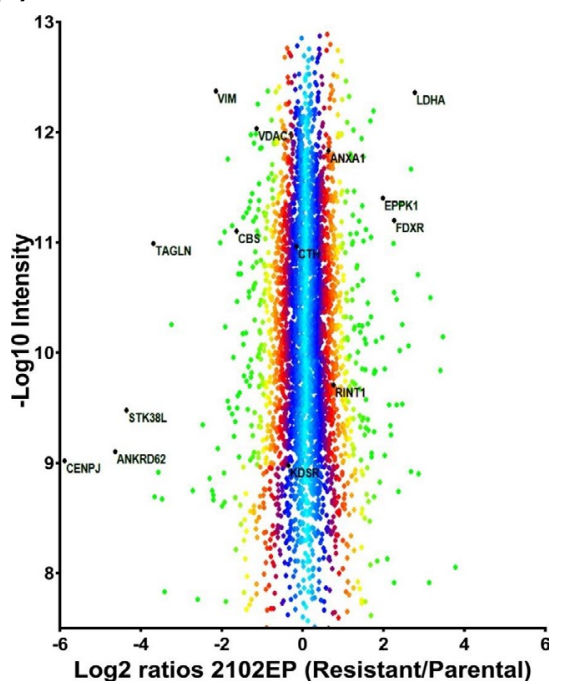

(d)

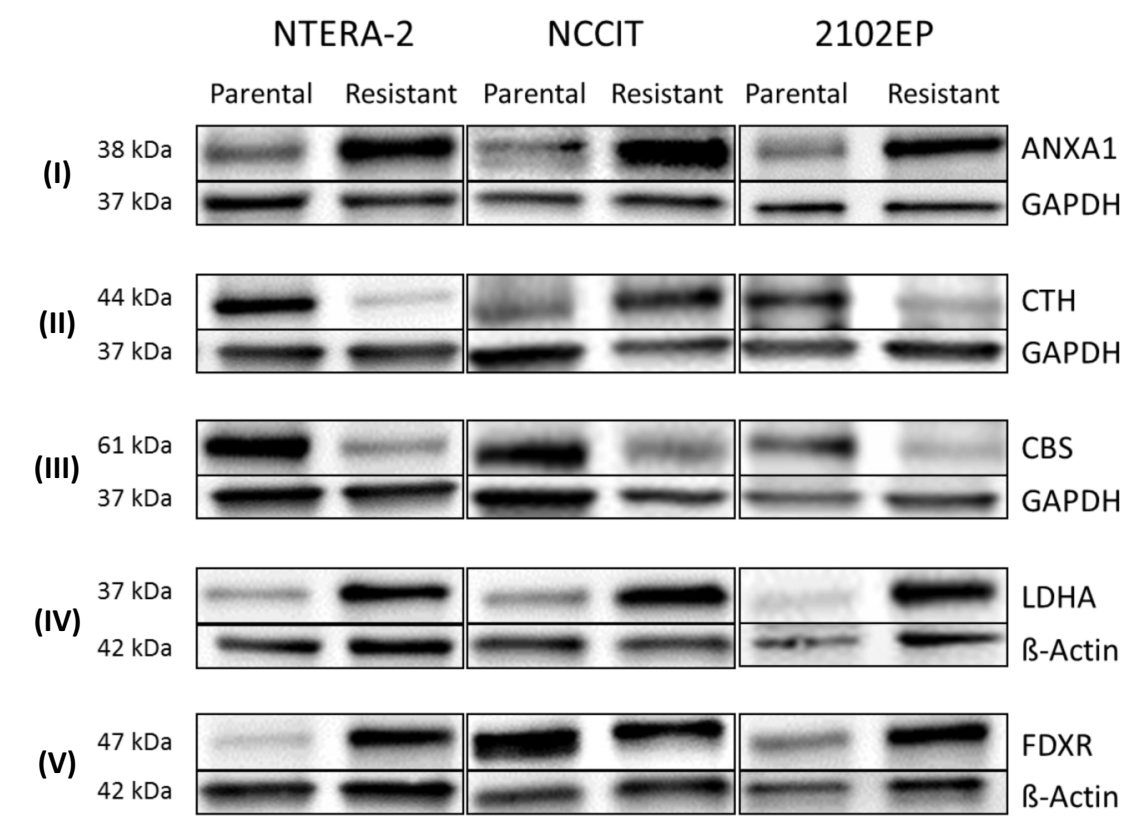

Fig. 2 Distribution of proteins from SILAC and validation of protein expression by Western Blot analysis. a-c Distribution of SILAC ratios of all quantified proteins according to their relative expression in the resistant vs. sensitive cells lines of NTERA-2, NCCIT and 2102EP. d CBS (I) is downregulated in NTERA-2-R, NCCIT-R and

\section{Western blot analysis}

We were able to confirm the obtained MS results using Western blotting of selected proteins described in Table 1 (see Fig. 2d). The selected proteins showed a significant different regulation in at least two or all three cell lines in comparison to the parental lineages (Table 1). All three
2102EP-R. ANXA1 (II) and LDHA (III) are upregulated in NTERA2-R, NCCIT-R and 2102EP-R. FDXR (IV) is upregulated in NTERA2-R and 2102EP-R with similar expression in NCCIT and NCCIT-R. CTH (V) is downregulated in NTERA-2-R and 2102EP and shows higher expression in NCCIT-R

cisplatin-resistant cell lines showed lower expression of cystathionine beta-synthase (CBS) and cystathionine gamma-lyase (CTH) levels were lower in NTERA-2-R and 2102EP-R compared with the cisplatin-sensitive counterpart (Fig. 2d I and II). Amounts of Annexin A1 (ANXA1), the L-lactate dehydrogenase A (LDHA) and NADPH-adrenodoxin oxidoreductase (FDXR) were increased in all three 
Table 1 Overlapping proteins possibly associated with cisplatin resistance

\begin{tabular}{|c|c|c|c|c|c|c|c|}
\hline Protein name & Gene name & NTERA-2 (R/S) & $p$ value & $\operatorname{NCCIT}(\mathrm{R} / \mathrm{S})$ & $p$ value & 2102EP (R/S) & $p$ value \\
\hline Cystathionine beta-synthase & CBS & $0.24 \downarrow$ & $2.38 \mathrm{E}-06$ & $0.44 \downarrow$ & $4.52 \mathrm{E}-04$ & $0.34 \downarrow$ & $2.20 \mathrm{E}-04$ \\
\hline Annexin A1 & ANXA1 & $4.89 \uparrow$ & $3.13 \mathrm{E}-10$ & $3.14 \uparrow$ & $2.46 \mathrm{E}-06$ & $1.44 \uparrow$ & $1.98 \mathrm{E}-01$ \\
\hline Rabenosyn-5 & RBSN & $34.36 \uparrow$ & $3.16 \mathrm{E}-09$ & $35.74 \uparrow$ & $1.78 \mathrm{E}-14$ & $\mathrm{NaN}$ & $\mathrm{NaN}$ \\
\hline L-lactate dehydrogenase A chain & LDHA & $4.10 \uparrow$ & 2.29E-08 & $2.18 \uparrow$ & $1.35 \mathrm{E}-03$ & $5.47 \uparrow$ & $5.79 \mathrm{E}-10$ \\
\hline $\begin{array}{l}\text { NADPH:adrenodoxin oxidoreductase, mitochon- } \\
\text { drial }\end{array}$ & FDXR & $8.59 \uparrow$ & $3.18 \mathrm{E}-13$ & $1.27 \uparrow$ & 4.72E-01 & $3.96 \uparrow$ & $1.63 \mathrm{E}-07$ \\
\hline Epiplakin & EPPK1 & $3.24 \uparrow$ & $3.48 \mathrm{E}-06$ & $0.58 \downarrow$ & 1.19E-02 & $3.31 \uparrow$ & $1.33 \mathrm{E}-05$ \\
\hline Ankyrin repeat domain-containing protein 62 & ANKRD62 & $5.22 \uparrow$ & 9.97E-03 & $53.86 \uparrow$ & $1.51 \mathrm{E}-18$ & $0.05 \downarrow$ & $2.41 \mathrm{E}-18$ \\
\hline Vimentin & VIM & $1.60 \uparrow$ & $6.77 \mathrm{E}-02$ & $2.70 \uparrow$ & $4.32 \mathrm{E}-05$ & $0.25 \downarrow$ & $5.46 \mathrm{E}-10$ \\
\hline $\begin{array}{l}\text { Voltage-dependent anion-selective channel } \\
\text { protein } 1\end{array}$ & VDAC1 & $1.30 \uparrow$ & $3.26 \mathrm{E}-01$ & $2.34 \uparrow$ & $4.78 \mathrm{E}-04$ & $0.47 \downarrow$ & $6.49 \mathrm{E}-04$ \\
\hline Collagen alpha-1(I) chain & COL1A1 & $210.43 \uparrow$ & $1.37 \mathrm{E}-56$ & $0.11 \downarrow$ & $3.20 \mathrm{E}-12$ & $\mathrm{NaN}$ & $\mathrm{NaN}$ \\
\hline Cystathionine gamma-lyase & $\mathrm{CTH}$ & $0.31 \downarrow$ & $1.02 \mathrm{E}-04$ & $0.35 \downarrow$ & 7.14E-06 & $0.87 \downarrow$ & $5.87 \mathrm{E}-01$ \\
\hline 3-ketodihydrosphingosine reductase & KDSR & $0.20 \downarrow$ & $2.02 \mathrm{E}-04$ & $12.86 \uparrow$ & $2.25 \mathrm{E}-08$ & $0.77 \downarrow$ & $5.43 \mathrm{E}-01$ \\
\hline RAD50-interacting protein 1 & RINT1 & $0.23 \downarrow$ & $6.19 \mathrm{E}-05$ & $13.42 \uparrow$ & $1.29 \mathrm{E}-10$ & $1.55 \uparrow$ & $1.84 \mathrm{E}-01$ \\
\hline Transgelin & TAGLN & $3.20 \uparrow$ & $1.12 \mathrm{E}-04$ & $\mathrm{NaN}$ & $\mathrm{NaN}$ & $0.09 \downarrow$ & $7.36 \mathrm{E}-16$ \\
\hline Centromere protein $\mathrm{J}$ & CENPJ & $19.94 \uparrow$ & 7.19E-07 & $1.21 \uparrow$ & 7.47E-01 & $0.02 \downarrow$ & $2.21 \mathrm{E}-28$ \\
\hline Serine/threonine-protein kinase 38-like & STK38L & $12.34 \uparrow$ & $1.06 \mathrm{E}-06$ & $0.70 \downarrow$ & $3.04 \mathrm{E}-01$ & $0.06 \downarrow$ & $2.05 \mathrm{E}-16$ \\
\hline
\end{tabular}

The table summarizes the protein ratios in cisplatin-resistant cell lines (R) compared with the sensitive parental control cell lines (S) and the corresponding $p$ values of all proteins that showed significantly different levels in at least two of the three tested cell lines. Increased levels are is highlighted with a green arrow $(\uparrow)$ and decreased levels are marked with a red arrow ( $\downarrow)$

NaN not a number

resistant cell lines (Fig. 2d III-V), which is in accordance with the mass spectrometric analysis results (Fig. 2a-c).

\section{Gene set enrichment analysis}

In order to gain functional insights into the mechanisms of cisplatin resistance, we performed a gene set enrichment analysis (GSEA) of all genes coding for proteins altered in each cell line individually or commonly. GSEA determines, if a defined set of genes shows a statistically significant difference in enrichment between two biological states (cisplatin resistance vs. sensitivity) [21, 22]. We compared the cisplatin-resistance ratios to 50 hallmark gene sets representing specific biological states or processes (http://www.gsea$\mathrm{msigdb}$.org/gsea/msigdb/collections.jsp). In each cisplatinresistant cell line individually, we found a heterogenous set of processes affected. The analysis of NTERA-2-R detected 'Interferon alpha and gamma' signalling and 'epithelial to mesenchymal transition' (EMT) as mainly enriched gene sets (Supplementary Fig. 2a-d). Two sets related to 'Myc targeting' and 'DNA repair' were significantly affected in NCCIT-R cell line (Supplementary Fig. 2e-h). Furthermore, GSEA revealed seven significantly affected gene sets including 'P53 signalling', 'hypoxia', 'fatty acid metabolism', 'glycolysis', 'late estrogen response', 'oxidative phosphorylation' and 'IL2 STAT5 signalling' for 2102EP-R (Supplementary Fig. 2i-p). The combined analysis of the three cell lines highlighted 'DNA repair', 'oxidative phosphorylation' and 'early estrogen response' as the three most prominently enriched gene sets (Fig. 3a-c).

\section{STRING interaction prediction, DAVID gene ontology and TCGA cohort analysis}

First, the DAVID Gene ontology tool was used to predict molecular functions of all proteins altered in the resistant cell lines (Supplementary Fig. 3 a, c, e, g, i, k). Proteins increased in amount in the resistant cells compared with the parental cell lines were involved in oxidoreductase activity, acetylation and metal binding were among others commonly deregulated in all three cell lines. Next, the STRING algorithm was used to predict protein-protein interactions of the enriched proteins (Supplementary Fig. 3b, d, f). Proteins involved in the molecular processes found by the DAVID analysis were highlighted by colour. In contrast, proteins decreased in amount in resistant cell lines compared with the parental cell lines were involved in phosphatidyl-inositol binding and DNA methyltransferase activity (Supplementary Fig. 3 g-1).

Furthermore, using cBioPortal, we screened the TCGA GCT tissue cohort for mutational footprints and expression of all genes coding for affected proteins in resistant cell lines individually (Supplementary Fig. 4). In general, 149 GCT (seminoma and nonseminoma) samples were included. The 
Fig. 3 GSEA analysis reveals DNA repair as common gene set deregulated in cisplatin-resistant cells. a The ten most affected gene sets of the combined ratios of three NT2, four NCCIT and four 2102EP replicates of proteomic analysis represented as the $-\log 2$ of the nominal (NOM) $q$ value and the FDR $q$ value. b Enrichment plot: DNA REPAIR Profile of the Running ES Score and Positions of GeneSet Members on the Rank Ordered List $(p<0.004$, $q<0.19)$. c Blue-Pink O' Gram in the Space of the analyzed GeneSet representing the ratio values of all genes in the GeneSet referred to 0

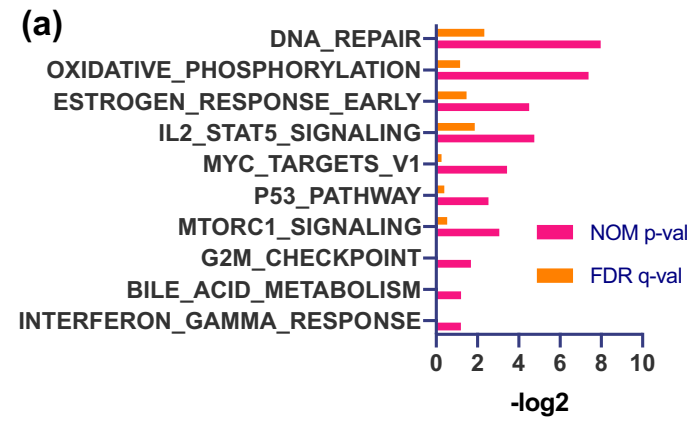

(b)

(c)

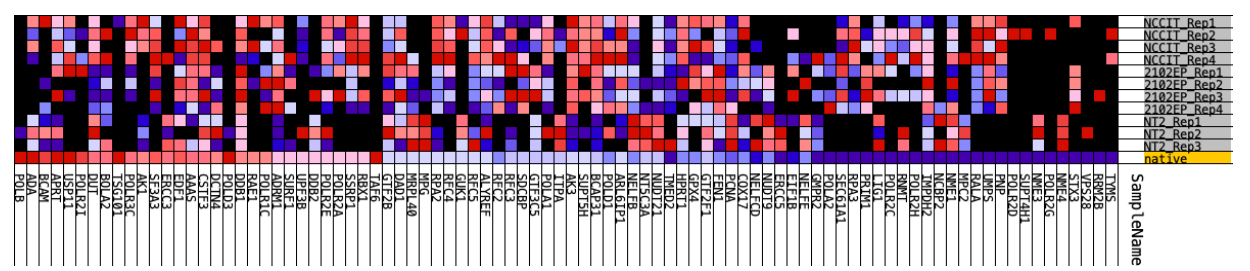

majority of the samples were highly aneuploid, harbored a 12gain, were mainly in the typical age of 14-44 years and were isolated from testis. Indeed, expression of analyzed genes was mainly associated with a nonseminomatous cell character (Supplementary Fig. 4). Nevertheless, expression of a subset of genes could also be related to seminomas. Mutations in the analyzed genes were overall rare, with IFITM3 (enriched in 2102EP-R) harboring a deep deletion in $2.1 \%$ of samples, GDF3 being amplified in $7 \%$ (enriched in NCCIT-R) and STK38L (enriched in NT2/D1-R) being amplified in $8 \%$ as most affected genes by mutation.

\section{Discussion}

Cisplatin resistance in GCTs rarely occurs in primary diseases, but in up to $15 \%$ of metastatic diseases with poor prognosis for the affected patient [5]. The mechanisms of cisplatin resistance in GCTs are complex, still not completely understood and might depend on GCT subtypes [27]. Different cellular and molecular mechanisms and GCT characteristics, such as DNA damage repair systems, the p53/MDM2 axis and apoptotic pathways as well as epigenetic changes have been investigated and seem to contribute to cisplatin resistance as a multifactorial phenomenon [27]. Because tissue specimens from cisplatin-resistant GCTs are rare, we analyzed the protein expression profiles of three different cisplatin-resistant GCT cell lines (NTERA-2-R, NCCIT-R and 2102EP-R) and their cisplatin-sensitive parental counterparts using mass spectrometry in combination with stable isotope labelling with amino acids in cell culture (SILAC) $[13,14]$ to detect proteins with a possible impact on cisplatin resistance. In addition, GSEA, DAVID Gene Ontology and STRING analyses were performed and the results were compared with the TCGA GCT tissue cohort.

Cystathionine beta-synthase (CBS) was significantly decreased in amount in all three cisplatin-resistant cell lines in comparison to their cisplatin-sensitive parental lineage. CBS is an enzyme that regulates homocysteine metabolism and catalyzes the formation of cystathionine [28]. Furthermore, it participates in different desulfurization reactions, which lead to the production of hydrogen sulfide $\left(\mathrm{H}_{2} \mathrm{~S}\right)$ [29]. CBS is most commonly synthesized in the liver, the pancreas, the kidney and the brain with only low baseline expression in testicular tissues [30, 31]. Its role in cancer biology is complex and seems to be cancer type-specific [30]. For example, serous ovarian cancer [32] as well as invasive urothelial bladder carcinoma [33], colorectal cancers [27] and prostate cancer [28] have shown high levels of CBS and inhibiting CBS has improved the effect of cisplatin-based chemotherapy in these neoplasms. The downregulation of CBS via small molecule inhibitors or siRNAs reduced antioxidant capacity and therefore enhanced the sensitivity of cancer cells to chemotherapy. It is also suggested that decreased levels of CBS and $\mathrm{H}_{2} \mathrm{~S}$ production might activate the intrinsic apoptotic pathway via release of mitochondrial cytochrom C [34]. According to the human protein atlas [35], testicular GCT tissue show low synthesis of CBS and other molecules of sulfur metabolism (e.g. glutathione (GSH) or methallothioneins). Masters et al. also showed, that cisplatin-sensitive GCT cell lines have low levels of GSH and glutathione-S-transferase (GST) [36]. These findings are not quite in accordance with our findings of low CBS levels in cisplatin-resistant GCT cell lines, but further investigations are required to better define its role in resistance. 
Another enzyme of the cysteine metabolism, namely cystathionine gamma lyase (CTH), showed decreased levels in two of the three resistant cell lines (NTERA-2-R and NCCIT-R). CTH catalyzes the breakdown of cystathionine to cysteine, alpha-ketobutyrate and $\mathrm{H}_{2} \mathrm{~S}$. It plays a key role in the bodies $\mathrm{H}_{2} \mathrm{~S}$ production [28, 30]. Its function in tumour biology has only been investigated in some tumour entities. The studies showed, similar to the role of CBS in the abovementioned tumours, that murine prostate cancer cells showed high amounts of CTH and its product $\mathrm{H}_{2} \mathrm{~S}$ in metastatic prostate cancer [37], similar to breast cancer cells $[38,39]$. Inhibition of CTH resulted in a decreased tumour burden. In our study, resistant NTERA-2 and NCCIT showed decreased levels of CTH in comparison to the sensitive cell lines. A function of low CTH levels in resistant tumour cell lines remains to be elucidated, yet.

The Annexin A1 (ANXA1) levels were significantly increased in resistant NTERA-2 and NCCIT cell lines. ANXA1 is a calcium-dependent phospholipid binding protein that belongs to the Annexin superfamily [40-42]. ANXA1 is a substrate for different kinases, e.g. epidermal growth factor receptor kinase, and is involved in different cellular pathways which are in association with inflammation, cell differentiation and proliferation [43]. Its role in tumour biology (e.g. tumour development, proliferation) has been conflicting because its expression is increased in some cancers (e.g. esophageal, gastric, colorectal, pancreatic and lung adenocarcinoma) and decreased in others (e.g. esophageal, lung squamous cell carcinoma, breast carcinoma and prostatic adenocarcinoma), but a common function of ANXA1 in chemosensitivity was reported [44-46]. Wang et al. could show an increased ANXA1 expression in a platin-resistant cell line of pulmonary adenocarcinoma and verified this in primary tumour tissue of cisplatin-resistant patients [47]. These previously reported roles of ANXA1 complement our findings and supports the contribution of ANXA1 to the development of cisplatin resistance irrespective of the tumours' tissues of origin.

In addition, the protein levels of L-lactate dehydrogenase A chain (LDHA) were elevated in NTERA-2-R and 2102EP-R cells. LDHA plays an important role in anaerobic glycolysis [48, 49]. In tumour cells, LDHA plays essential role in initiation, growth, tumour maintenance, progression and metastasis [49]. Increased LDH levels are often used as diagnostic markers, prognostic factors and indicators of treatment response for GCTs and other tumours [50]. According to cisplatin treatment response, Manerba et al. showed that inhibition of LDH in Burkitt's lymphoma cells increased cisplatin sensitivity possibly through higher amounts of reactive oxygen species (ROS) [51]. This can on the opposite indicate that higher levels of LDHA in cisplatin-resistant GCT cell lines help to overcome cell stress.
However, further investigations regarding the detailed role of LDHA in cisplatin-resistant cell lines are necessary.

Furthermore, a significantly increase of COL1A1 and COL1A2 levels was detected in cisplatin-resistant NTERA-2 cell lines in comparison with the parental cell line, with decrease in resistant NCCIT cell line. Both proteins resemble extracellular matrix proteins for that increased levels have been detected in stressed cells [52]. They have further been shown to play an important role in cisplatin-resistance in pulmonary adenocarcinoma cell lines [52]. It remains unclear why the protein levels are different in the three different cell lines and needs further investigation.

GSEA revealed that DNA repair is the only significant gene set deregulated in all three cell lines, which has been considered for cisplatin resistance in GCT before [27]. In NTERA-2-R, interferon alpha and gamma signalling as well as epithelial to mesenchymal transition (EMT) were the most enriched gene sets. The role of EMT in chemoresistance has been described by Ashrafizadeh et al. but has not been evaluated for GCTs [53]. NCCIT cell lines showed significantly deregulated sets of MYC targeting and DNA repair. The function of DNA repair mechanisms in GCT cisplatin resistance are still slightly controversial, since both, upregulation and downregulation of DNA repair-associated genes has been described in GCTs [27]. For 2102EP cells, GSEA revealed seven significantly affected gene sets, including p53 signalling, oxidative phosphorylation and IL2 STAT5 signalling. Mutations and inactivation of p53 are known to be involved in cisplatin resistance of GCT by reducing apoptotic cell death [54].

DAVID Gene Ontology and STRING analyses showed a deregulation of proteins involved in oxidoreductase activity, acetylation and metal binding. The upregulation of NADPHadrenodoxin oxidoreductase (FDXR) in resistant cell lines could be validated by Western blot. Oxidoreductase activity, acetylation and metal binding have been described in connection to p53 [55-57]. A reduced p53 activity has been shown to induce an increased resistance against cisplatin in testicular germ cell-derived human embryonal carcinoma cells also by a direct regulation of FDRX [58]. Although p53 pathway was only detected as significantly regulated in cisplatin-resistant 2102EP, these data indicate a strong p53 dependency and a deregulated oxidative stress response in cisplatin-resistant GCT.

Screening of the TCGA GCT cohort demonstrated that most genes/proteins found increased in the resistant situation, show already basal expression in GCT tissues (mostly nonseminomas). Thus, rather an overshooting/upregulation of expression than an induction of gene expression is associated with acquisition of therapy resistance. The mutational burden was overall low, nevertheless some genes were frequently mutated in GCT tissues, putatively affecting their molecular function. During development of cisplatin 
resistance, these mutated genes might be induced as well, further contributing to cisplatin resistance by their altered function or increased expression turnover.

In summary, high resolution mass spectrometry in combination with SILAC quantification is a powerful tool to detect differences of protein levels in cisplatin-resistant and cisplatin-sensitive cell lines. We detected 144 significantly deregulated proteins were found in cisplatin-resistant GCT cell lines. The findings of mass spectrometry could be validated by Western blot analysis. With this study, we therefore provide a large proteomic resource in vitro library for studying proteomic alterations contributing to acquired cisplatin resistance in testicular germ cell tumour cell lines. The detected and analysed proteins need further investigations to unravel their putative role in cisplatin resistance of GCT and to determine possible new treatment approaches.

Supplementary Information The online version contains supplementary material available at https://doi.org/10.1007/s00345-022-03936-1.

Author contributions All authors contributed to the study conception and design and FB supervised the project. Material preparation, data collection and/or analysis were performed by AF, HB, OE, AR, CL, PS, CO, SK, DN and FB. The first draft of the manuscript was written by $\mathrm{AF}, \mathrm{HB}, \mathrm{SK}, \mathrm{DN}$ and $\mathrm{FB}$ and all authors commented on previous versions of the manuscript. All authors read and approved the final manuscript. AF, HB, SK, DN and FB contributed equally to this work.

Funding Open Access funding enabled and organized by Projekt DEAL. F.B. is supported by the Wilhelm-Sander-Stiftung (Grant Nos. 2016.041.1, 2016.041.2, 2016.041.3). H.B. and O.E. are supported by Deutsche Krebshilfe Foundation (Grant No. 70112551). H.B. is supported by the University Medical Center Göttingen and the Else-Kröner-Fresenius-Stiftung.

Availability of data and material The mass spectrometry proteomics data have been deposited to the ProteomeXchange Consortium via the PRIDE [59] partner repository with the dataset identifier PXD030251. All other data is available on request from the corresponding author.

Code availability Not applicable.

\section{Declarations}

Conflict of interest The authors declare that they have no conflict of interest.

Ethics approval Not applicable.

Consent to participate Not applicable.

Consent for publication All authors agreed for publication of the study.

Open Access This article is licensed under a Creative Commons Attribution 4.0 International License, which permits use, sharing, adaptation, distribution and reproduction in any medium or format, as long as you give appropriate credit to the original author(s) and the source, provide a link to the Creative Commons licence, and indicate if changes were made. The images or other third party material in this article are included in the article's Creative Commons licence, unless indicated otherwise in a credit line to the material. If material is not included in the article's Creative Commons licence and your intended use is not permitted by statutory regulation or exceeds the permitted use, you will need to obtain permission directly from the copyright holder. To view a copy of this licence, visit http://creativecommons.org/licenses/by/4.0/.

\section{References}

1. Beyer J, Albers P, Altena R et al (2013) Maintaining success, reducing treatment burden, focusing on survivorship: highlights from the third European consensus conference on diagnosis and treatment of germ-cell cancer. Ann Oncol 24:878-888. https:// doi.org/10.1093/annonc/mds579

2. Oosterhuis JW, Looijenga LHJ (2005) Testicular germ-cell tumours in a broader perspective. Nat Rev Cancer 5:210-222. https://doi.org/10.1038/nrc1568

3. Moch H, Cubilla AL, Humphrey PA et al (2016) The 2016 WHO classification of tumours of the urinary system and male genital organs-part A: renal, penile, and testicular tumours. Eur Urol 70:93-105. https://doi.org/10.1016/j.eururo.2016.02.029

4. Honecker F, Aparicio J, Berney D et al (2018) ESMO consensus conference on testicular germ cell cancer: diagnosis, treatment and follow-up. Ann Oncol 29:1658-1686. https://doi.org/10. 1093/annonc/mdy217

5. Oing C, Seidel C, Bokemeyer C (2018) Therapeutic approaches for refractory germ cell cancer. Expert Rev Anticancer Ther 18:389-397. https://doi.org/10.1080/14737140.2018.1450630

6. Korkola JE, Houldsworth J, Bosl GJ, Chaganti RSK (2009) Molecular events in germ cell tumours: linking chromosome-12 gain, acquisition of pluripotency and response to cisplatin. BJU Int 104:1334-1338. https://doi.org/10.1111/j.1464-410X.2009. 08855.x

7. Moul JW, Dodge RK, Robertson JE et al (1991) The impact of the "cisplatin era" of treatment on survival in testicular cancer. World J Urol 9:45-50. https://doi.org/10.1007/BF00184714

8. Bokemeyer C, Oechsle K, Honecker F et al (2008) Combination chemotherapy with gemcitabine, oxaliplatin, and paclitaxel in patients with cisplatin-refractory or multiply relapsed germ-cell tumors: a study of the German testicular cancer study group. Ann Oncol 19:448-453. https://doi.org/10.1093/annonc/ mdm526

9. Feldman DR, Bosl GJ, Sheinfeld J, Motzer RJ (2008) Medical treatment of advanced testicular cancer. JAMA 299:672-684. https://doi.org/10.1001/jama.299.6.672

10. Kvammen $\varnothing$, Myklebust TÅ, Solberg A et al (2016) Long-term relative survival after diagnosis of testicular germ cell tumor. Cancer Epidemiol Biomarkers Prev 25:773-779. https://doi.org/10. 1158/1055-9965.EPI-15-1153

11. International Prognostic Factors Study Group, Lorch A, Beyer J et al (2010) Prognostic factors in patients with metastatic germ cell tumors who experienced treatment failure with cisplatinbased first-line chemotherapy. J Clin Oncol 28:4906-4911. https:// doi.org/10.1200/JCO.2009.26.8128

12. Bokemeyer C, Kollmannsberger C, Harstrick A et al (1999) Treatment of patients with cisplatin-refractory testicular germ-cell cancer. German testicular cancer study group (GTCSG). Int J Cancer 83:848-851. https://doi.org/10.1002/(sici)1097-0215(19991210) 83:6\%3c848::aid-ijc29\%3e3.0.co;2-\#

13. Eini R, Stoop H, Gillis AJM et al (2014) Role of SOX2 in the etiology of embryonal carcinoma, based on analysis of the NCCIT and NT2 cell lines. PLoS ONE 9:e83585. https://doi.org/10.1371/ journal.pone. 0083585 
14. Josephson R, Ording CJ, Liu Y et al (2007) Qualification of embryonal carcinoma 2102Ep as a reference for human embryonic stem cell research. Stem Cells 25:437-446. https://doi.org/ 10.1634/stemcells.2006-0236

15. Bohnenberger H, Oellerich T, Engelke M et al (2011) Complex phosphorylation dynamics control the composition of the Syk interactome in B cells. Eur J Immunol 41:1550-1562. https://doi. org/10.1002/eji.201041326

16. Bohnenberger H, Kaderali L, Ströbel P et al (2018) Comparative proteomics reveals a diagnostic signature for pulmonary headand-neck cancer metastasis. EMBO Mol Med. https://doi.org/10. 15252/emmm.201708428

17. Bremmer F, Bohnenberger H, Küffer S et al (2019) Proteomic comparison of malignant human germ cell tumor cell lines. Dis Markers 2019:8298524. https://doi.org/10.1155/2019/8298524

18. Oellerich T, Bremes V, Neumann K et al (2011) The B-cell antigen receptor signals through a preformed transducer module of SLP65 and CIN85. EMBO J 30:3620-3634. https://doi.org/10. 1038/emboj.2011.251

19. Fenske AE, Glaesener S, Bokemeyer C et al (2012) Cisplatin resistance induced in germ cell tumour cells is due to reduced susceptibility towards cell death but not to altered DNA damage induction or repair. Cancer Lett 324:171-178. https://doi.org/10. 1016/j.canlet.2012.05.009

20. Cox J, Mann M (2008) MaxQuant enables high peptide identification rates, individualized p.p.b.-range mass accuracies and proteome-wide protein quantification. Nat Biotechnol 26:1367-1372. https://doi.org/10.1038/nbt.1511

21. Subramanian A, Tamayo P, Mootha VK et al (2005) Gene set enrichment analysis: a knowledge-based approach for interpreting genome-wide expression profiles. Proc Natl Acad Sci USA 102:15545-15550. https://doi.org/10.1073/pnas.0506580102

22. Mootha VK, Lindgren CM, Eriksson K-F et al (2003) PGC1alpha-responsive genes involved in oxidative phosphorylation are coordinately downregulated in human diabetes. Nat Genet 34:267-273. https://doi.org/10.1038/ng1180

23. Szklarczyk D, Gable AL, Lyon D et al (2019) STRING v11: protein-protein association networks with increased coverage, supporting functional discovery in genome-wide experimental datasets. Nucleic Acids Res 47:D607-D613. https://doi.org/10. 1093/nar/gky1131

24. Dennis G, Sherman BT, Hosack DA et al (2003) DAVID: database for annotation, visualization, and integrated discovery. Genome Biol 4:P3

25. Gao J, Aksoy BA, Dogrusoz U et al (2013) Integrative analysis of complex cancer genomics and clinical profiles using the cBioPortal. Sci Sig. https://doi.org/10.1126/scisignal.2004088

26. Cerami E, Gao J, Dogrusoz U et al (2012) The cBio cancer genomics portal: an open platform for exploring multidimensional cancer genomics data. Cancer Discov 2:401-404. https://doi.org/ 10.1158/2159-8290.CD-12-0095

27. Lobo J, Jerónimo C, Henrique R (2020) Cisplatin resistance in testicular germ cell tumors: current challenges from various perspectives. Cancers (Basel). https://doi.org/10.3390/cancers120 61601

28. Borcsok E, Abeles RH (1982) Mechanism of action of cystathionine synthase. Arch Biochem Biophys 213:695-707. https://doi. org/10.1016/0003-9861(82)90600-2

29. Szabo C, Coletta C, Chao C et al (2013) Tumor-derived hydrogen sulfide, produced by cystathionine- $\beta$-synthase, stimulates bioenergetics, cell proliferation, and angiogenesis in colon cancer. Proc Natl Acad Sci USA 110:12474-12479. https://doi.org/10.1073/ pnas. 1306241110
30. Zhu H, Blake S, Chan KT et al (2018) Cystathionine $\beta$-synthase in physiology and cancer. Biomed Res Int 2018:3205125. https:// doi.org/10.1155/2018/3205125

31. Tissue expression of CBS - summary - the human protein atlas. https://www.proteinatlas.org/ENSG00000160200-CBS/tissue. Accessed 23 Apr 2021

32. Kawahara B, Ramadoss S, Chaudhuri G et al (2019) Carbon monoxide sensitizes cisplatin-resistant ovarian cancer cell lines toward cisplatin via attenuation of levels of glutathione and nuclear metallothionein. J Inorg Biochem 191:29-39. https://doi.org/10. 1016/j.jinorgbio.2018.11.003

33. Wahafu W, Gai J, Song L et al (2018) Increased H2S and its synthases in urothelial cell carcinoma of the bladder, and enhanced cisplatin-induced apoptosis following H2S inhibition in EJ cells. Oncol Lett 15:8484-8490. https://doi.org/10.3892/ol.2018.8373

34. Pagliara V, Saide A, Mitidieri E et al (2016) 5-FU targets rpL3 to induce mitochondrial apoptosis via cystathionine- $\beta$-synthase in colon cancer cells lacking p53. Oncotarget 7:50333-50348. https://doi.org/10.18632/oncotarget.10385

35. Expression of CBS in testis cancer-the human protein atlas. https://www.proteinatlas.org/ENSG00000160200-CBS/patho logy/testis+cancer. Accessed 23 Apr 2021

36. Masters JR, Thomas R, Hall AG et al (1996) Sensitivity of testis tumour cells to chemotherapeutic drugs: role of detoxifying pathways. Eur J Cancer 32A:1248-1253. https://doi.org/10.1016/ 0959-8049(96)00033-0

37. Wang Y-H, Huang J-T, Chen W-L et al (2019) Dysregulation of cystathionine $\gamma$-lyase promotes prostate cancer progression and metastasis. EMBO Rep 20:e45986. https://doi.org/10.15252/embr. 201845986

38. Wang L, Shi H, Liu Y et al (2019) Cystathionine- $\gamma$-lyase promotes the metastasis of breast cancer via the VEGF signaling pathway. Int J Oncol 55:473-487. https://doi.org/10.3892/ijo.2019.4823

39. You J, Shi X, Liang H et al (2017) Cystathionine- $\gamma$-lyase promotes process of breast cancer in association with STAT3 signaling pathway. Oncotarget 8:65677-65686. https://doi.org/10. 18632/oncotarget.20057

40. Geisow MJ (1986) Common domain structure of Ca2+ and lipidbinding proteins. FEBS Lett 203:99-103. https://doi.org/10.1016/ 0014-5793(86)81445-4

41. Pepinsky RB, Tizard R, Mattaliano RJ et al (1988) Five distinct calcium and phospholipid binding proteins share homology with lipocortin I. J Biol Chem 263:10799-10811

42. Crumpton MJ, Dedman JR (1990) Protein terminology tangle. Nature 345:212-212. https://doi.org/10.1038/345212a0

43. Lim LHK, Pervaiz S (2007) Annexin 1: the new face of an old molecule. FASEB J 21:968-975. https://doi.org/10.1096/fj.067464rev

44. Foo SL, Yap G, Cui J, Lim LHK (2019) Annexin-A1—a blessing or a curse in cancer? Trends Mol Med 25:315-327. https://doi. org/10.1016/j.molmed.2019.02.004

45. Mussunoor S, Murray GI (2008) The role of annexins in tumour development and progression. J Pathol 216:131-140. https://doi. org/10.1002/path.2400

46. Elakad O, Li Y, Gieser N et al (2021) Role of annexin A1 in squamous cell lung cancer progression. Dis Markers 2021:e5520832. https://doi.org/10.1155/2021/5520832

47. Wang C, Xiao Q, Li Y-W et al (2014) Regulatory mechanisms of annexin-induced chemotherapy resistance in cisplatin resistant lung adenocarcinoma. Asian Pac J Cancer Prev 15:3191-3194. https://doi.org/10.7314/apjcp.2014.15.7.3191

48. Pineda JRET, Callender R, Schwartz SD (2007) Ligand binding and protein dynamics in lactate dehydrogenase. Biophys $\mathbf{J}$ 93:1474-1483. https://doi.org/10.1529/biophysj.107.106146 
49. Miao P, Sheng S, Sun X et al (2013) Lactate dehydrogenase a in cancer: a promising target for diagnosis and therapy. IUBMB Life 65:904-910. https://doi.org/10.1002/iub.1216

50. Dieckmann K-P, Simonsen-Richter H, Kulejewski M et al (2019) Serum tumour markers in testicular germ cell tumours: frequencies of elevated levels and extents of marker elevation are significantly associated with clinical parameters and with response to treatment. Biomed Res Int 2019:5030349. https://doi.org/10.1155/ 2019/5030349

51. Manerba M, Di Ianni L, Fiume L et al (2015) Lactate dehydrogenase inhibitors sensitize lymphoma cells to cisplatin without enhancing the drug effects on immortalized normal lymphocytes. Eur J Pharm Sci 74:95-102. https://doi.org/10.1016/j.ejps.2015. 04.022

52. Jia R, Wang C (2020) MiR-29b-3p reverses cisplatin resistance by targeting COL1A1 in non-small-cell lung cancer A549/DDP cells. Cancer Manag Res 12:2559-2566. https://doi.org/10.2147/ CMAR.S246625

53. Ashrafizadeh M, Zarrabi A, Hushmandi K et al (2020) Association of the epithelial-mesenchymal transition (EMT) with cisplatin resistance. Int J Mol Sci. https://doi.org/10.3390/ijms21114002

54. Houldsworth J, Xiao H, Murty V et al (1998) Human male germ cell tumor resistance to cisplatin is linked to TP53 gene mutation. Oncogene 16:2345-2349. https://doi.org/10.1038/sj.onc.1201770
55. Bykov VJN, Lambert JMR, Hainaut P, Wiman KG (2009) Mutant p53 rescue and modulation of p53 redox state. Cell Cycle 8:2509_ 2517. https://doi.org/10.4161/cc.8.16.9382

56. Kong X, Guan J, Li J et al (2017) P66Shc-SIRT1 regulation of oxidative stress protects against cardio-cerebral vascular disease. Mol Neurobiol 54:5277-5285. https://doi.org/10.1007/ s12035-016-0073-2

57. Phatak VM, Muller PAJ (2015) Metal toxicity and the p53 protein: an intimate relationship. Toxicol Res 4:576-591. https://doi.org/ 10.1039/C4TX00117F

58. Kerley-Hamilton JS, Pike AM, Li N et al (2005) A p53-dominant transcriptional response to cisplatin in testicular germ cell tumorderived human embyronal carcinoma. Oncogene 24:6090-6100. https://doi.org/10.1038/sj.onc.1208755

59. Perez-Riverol Y, Csordas A, Bai J et al (2019) The PRIDE database and related tools and resources in 2019: improving support for quantification data. Nucleic Acids Res 47:D442-D450. https:// doi.org/10.1093/nar/gky1106

Publisher's Note Springer Nature remains neutral with regard to jurisdictional claims in published maps and institutional affiliations.

\section{Authors and Affiliations}

\section{A. Fichtner ${ }^{1} \cdot$ H. Bohnenberger ${ }^{1} \cdot$ O. Elakad ${ }^{1} \cdot$ A. Richter ${ }^{1} \cdot$ C. Lenz ${ }^{2,3} \cdot$ C. Oing $^{4,5} \cdot$ P. Ströbel ${ }^{1} \cdot$ S. Kueffer ${ }^{1}$. D. Nettersheim ${ }^{6} \cdot$ F. Bremmer ${ }^{1}$ (B)}
A. Fichtner
alexander.fichtner@med.uni-goettingen.de
H. Bohnenberger
hanibal.bohnenberger@med.uni-goettingen.de
O. Elakad
omar.elakad@med.uni-goettingen.de

\section{A. Richter}

annika.richter1@med.uni-goettingen.de

C. Lenz

christof.lenz@med.uni-goettingen.de

C. Oing

c.oing@uke.de

P. Ströbel

philipp.stroebel@med.uni-goettingen.de

S. Kueffer

stefan.kueffer@med.uni-goettingen.de

D. Nettersheim

Daniel.Nettersheim@med.uni-duesseldorf.de
1 Institute of Pathology, University Medical Center, Robert-Koch-Str. 40, 37075 Göttingen, Germany

2 Department of Clinical Chemistry, University Medical Center, Göttingen, Germany

3 Bioanalytical Mass Spectrometry Group, Max Planck Institute for Biophysical Chemistry, Göttingen, Germany

4 Department of Oncology, Hematology and Bone Marrow Transplantation with Division of Pneumology, University Medical Center Hamburg-Eppendorf, Martinistraße 52, 20246 Hamburg, Germany

5 Mildred Scheel Cancer Career Center HaTriCS4, University Medical Center Eppendorf, Hamburg, Germany

6 Department of Urology, Urological Research Laboratory, Translational UroOncology, Medical Faculty and University Hospital Düsseldorf, Heinrich Heine University Düsseldorf, Universitätsstrasse 1, 40225 Düsseldorf, Germany 